\title{
Weibliche Genitalverstümmelung im Kontext der Migration: eine Herausforderung für medizinisches Fachpersonal
}

\section{Marisa Birri}

Fachfrau gender-based violence, TERRE DES FEMMES Schweiz

1 Mädchenbeschneidung in der Schweiz. Umfrage bei Schweizer Hebammen, Gynäkologen/-innen, Pädiatern/-innen und Sozialstellen. Schweizerisches Komitee für UNICEF;

2005. S. 5

$2 \mathrm{Vgl}$. die Statistik der Weltgesundheitsorganisation WHO: www.who.int/ reproductivehealth/topics/ fgm/prevalence/en/index. html

3 Eyer P, Schweizer R. Die somalische und die eritreische Diaspora in de Schweiz. Bern: Bundesamt für Migration (Hrsg.); 2010, S. $42 \mathrm{f}$.

4 Ebd. S. 78

5 Mädchenbeschneidung in der Schweiz. Umfrage bei Schweizer Hebammen, Gynäkologen/-innen, Pädiatern/-innen und Sozialstellen. Schweizerisches Komitee für UNICEF; 2005. S. 9

Korrespondenz:

Marisa Birri

Standstrasse 32

CH-3014 Bern

Tel. 0313113879

info@terre-des-femmes.ch
Weltweit sind gemäss Schätzungen der Weltgesundheitsorganisation (WHO) zwischen 100 und 150 Millionen Mädchen und Frauen genital verstümmelt. Jedes Jahr kommen 3 Millionen dazu. Female Genital Mutilation (FGM) kommt rund um den Globus vor sehr stark verbreitet ist FGM aber im westlichen und östlichen Afrika, in einigen Ländern Asiens sowie im Mittleren Osten. Durch Migration ist weibliche Genitalverstümmelung auch in Europa, Nordamerika und Australien ein Thema. Die Begründungen für FGM sind vielfältig und je nach Gruppe und Region unterschiedlich: Die Beschneidung ist sehr oft eine Bedingung für die Heiratsfähigkeit der Frau. FGM soll die Sexualität der Frau kontrollieren und so die Jungfräulichkeit vor der Ehe und Treue während der Ehe garantieren. FGM wird aber auch mit Hygiene und Ästhetik begründet. Für viele ist FGM zudem ein Initiationsritus, bei dem das Mädchen zur Frau wird. Häufig werden auch religiöse Gründe genannt, obwohl keine Religion FGM vorschreibt.

FGM ist eine gravierende Menschenrechtsverletzung an Mädchen und Frauen. Sie ist Ausdruck einer tief in den jeweiligen sozialen, wirtschaftlichen und politischen Strukturen verwurzelten Ungleichheit der Geschlechter und stellt eine extreme Form der Diskriminierung von Frauen dar. FGM verletzt $\mathrm{u}$.a. das Recht auf Leben, das Recht auf Gesundheit und das Recht auf physische und psychische Integrität.

\section{Migrationshintergrund nicht ausser acht lassen}

In der Schweiz sind gemäss Schätzungen bis zu 7000 Mädchen und Frauen von FGM betroffen oder gefährdet. Die häufigsten Ursprungsländer dieser Frauen in der Schweiz sind Somalia, Äthiopien und Eritrea [1]. Dies spiegelt die Vorkommensrate in diesen Ländern und die Zahl der in der Schweiz lebenden Migrantinnen wider. In Somalia sind schätzungsweise $98 \%$ der Frauen von FGM betroffen, in Eritrea $89 \%$ und in Äthiopien 74\% [2].

Für Fachpersonen, die mit betroffenen Frauen in Kontakt kommen, ist es wichtig, den Migrationshintergrund dieser Personen zu berücksichtigen: Sehr oft stehen andere Probleme als FGM im Vordergrund, wie die Bewältigung von traumatischen Erfahrungen, schwierige sozioökonomische Bedingungen, Erwerbslosigkeit, das Zurechtfinden in einer fremden Umge-

\section{Mutilations génitales féminines}

Toutes les douze secondes, une jeune fille est victime d'une mutilation génitale, soit 100 à 150 millions de jeunes femmes touchées dans le monde entier. Avec la migration croissante - principalement du continent africain - les mutilations génitales féminines font également débat en Suisse. Elles représentent un nouveau défi que doivent relever les sagesfemmes, les médecins et le personnel soignant. L'objectif de cet article est d'exposer la problématique des mutilations génitales féminines dans le contexte de la migration en Suisse et de préciser les exigences que cela pose au personnel médical spécialisé.

bung, das Fehlen der unterstützenden Grossfamilie, Sprachprobleme sowie ein unsicherer Aufenthaltsstatus. Die überwiegende Mehrheit von Personen aus den am häufigsten von FGM betroffenen Gemeinschaften hat einen unsicheren Aufenthaltsstatus, d. h. befindet sich im Asylverfahren oder ist vorläufig aufgenommen [3]. Viele Migrantinnen und Migranten sind mit dem schweizerischen Gesundheitssystem nicht vertraut, und der Zugang dazu ist aufgrund von Sprachbarrieren und kulturell bedingten Hemmschwellen oft erschwert. All dies kann sich auf das physische und psychische Wohlbefinden dieser Personen negativ auswirken [4].

FGM kann deshalb nicht isoliert angegangen werden, sondern muss einhergehen mit Massnahmen, die auf ein «empowerment» der Migrantinnen, auf eine Verbesserung ihrer allgemeinen Lebenssituation, zielen.

\section{Was bedeutet dies für das medizinische Fachpersonal?}

Dieser Hintergrund stellt das medizinische Fachpersonal in der Schweiz vor grosse Herausforderungen. Mindestens jeder siebte Gynäkologe bzw. jede siebte Gynäkologin und jede siebte Hebamme in der 
FGM-Expertin aus Somalia in der Schweiz Die Somalierin Edna Adan Ismail, Gründerin und Leiterin der einzigen somaliländischen Geburtsklinik, des «Edna Adan Maternity Hospital», ist eine vielfach ausgezeichnete Pionierin im Kampf gegen die weibliche Genitalverstümmelung in Afrika. Die 73-Jährige wird vom 25. November bis zum 10. Dezember 2010 gemeinsam mit den Fachfrauen von TERRE DES FEMMES Schweiz verschiedene Veranstaltungen für Migrantinnen, Fachpersonal aus den Bereichen Migration und Gesundheit sowie für Mitarbeiter von Asylbehörden leiten. Auch die interessierte Öffentlichkeit erhält in mehreren öffentlichen Podien die Gelegenheit, diese eindrückliche Persönlichkeit kennenzulernen. Weitere Informationen: www. terre-des-femmes.ch.

6 Ebd. S. 5. Infibulation: Entfernung der Klitoris, Schamlippen und angrenzenden Teile und Zunähen bis auf eine kleine Öffnung. Exzision: Entfernung der Klitoris und der kleinen Schamlippen.

7 Thierfelder C. Female Genital Mutilation and the Swiss Health Care System. 2003; S. 39f.

8 Vgl. hierzu WHO, Female genital mutilation: integrating the prevention and the management of the health complications into the curricula of nursing and midwifery. A teacher's guide; 2001.

9 Siehe dazu die Guidelines der Schweizerischen Gesellschaft für Gynäkologie und Geburtshilfe: Patientinnen mit genitaler Beschneidung: Schweizerische Empfehlungen für Ärztinnen und Ärzte, Hebammen und Pflegefachkräfte; 2005. http://sggg.ch/ files/Patientinnen_mit_ genitaler_Beschneidung.pdf

10 Thierfelder C. Female Genital Mutilation and the Swiss Health Care System. 2003; S. XVII.

11 Eyer P, Schweizer R. Die somalische und die eritreische Diaspora in der Schweiz. Bern: Bundesamt für Migration (Hrsg.); 2010, S. 81.

12 Die Broschüre ist erhältlich in Deutsch, Französisch, Englisch, Amharisch, Arabisch, Somali und Tigrinya. Bestellung: www.terre-des-femmes.ch oder www.migesplus.ch

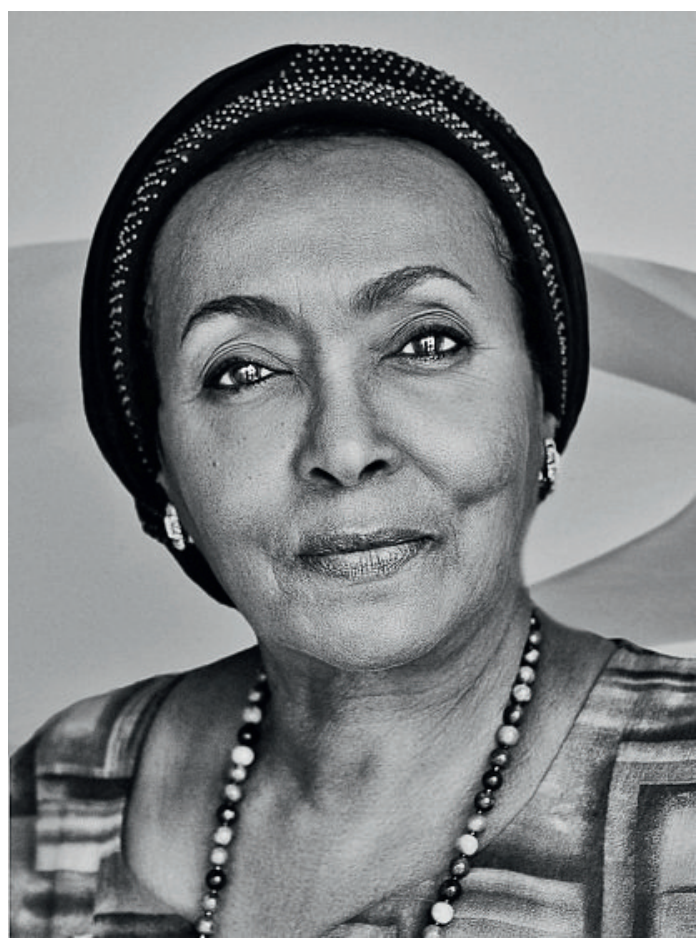

Pionierin im Kampf gegen die weibliche Genitalverstümmelung: Edna Adan.

Schweiz wurden schon mit einer beschnittenen Frau konfrontiert [5]. Die häufigsten Formen, die von den medizinischen Fachpersonen beobachtet wurden, waren Infibulation und Exzision [6]. Die betroffenen Frauen haben spezifische körperliche Probleme und Bedürfnisse, die eine spezifische und angemessene Behandlung verlangen. Schmerzhafte und verlängerte Menstruationsblutungen und Sexualstörungen werden von den betroffenen Frauen in der Schweiz als Hauptkomplikationen angegeben. In der Diaspora treten aber v.a. auch die psychosexuellen Aspekte der
Beschneidung in den Vordergrund. Häufig ist das ärztliche Gespräch in Bezug auf FGM unvollständig, besonders die psychosozialen und sexuellen Komplikationen werden selten angesprochen [7].

$\mathrm{Ob}$ die Frauen eine angemessene Behandlung erhalten, hängt vom Wissen und der Erfahrung der medizinischen Fachperson ab. Leider sind viele immer noch ungenügend sensibilisiert, und der Informationsbedarf bleibt gross. Das Thema muss deshalb dringend in das Curriculum der jeweiligen medizinischen Aus- und Weiterbildung integriert werden [8].

Dabei liegt die grösste Herausforderung weniger in der technischen Handhabung von Komplikationen von FGM [9] als in einer kultursensiblen, spezifischen Kommunikation und Interaktion zwischen dem medizinischen Personal und der Patientin. Oft sind die Sprachbarrieren, die Intimität des Themas und die Geschlechterrollen die Haupthindernisse [10]. Verständigungsschwierigkeiten und kulturell unterschiedliche Gesundheitsvorstellungen können

\section{Es braucht transkulturelle Kompetenzen und eine entsprechende Sensibilisierung}

die Kommunikation erschweren. So wünschen sich viele von FGM betroffene Frauen, von weiblichem medizinischem Personal betreut zu werden. Dies wird insbesondere von Personen aus der islamischen Glaubensgemeinschaft als starkes Bedürfnis geäussert. Es braucht also transkulturelle Kompetenzen und eine entsprechende Sensibilisierung auf die spezifischen Gesundheitsprobleme und Bedürfnisse der Migrationsbevölkerung [11].

Abgesehen von der Notwendigkeit einer angemessenen Behandlung der betroffenen Frauen und Mädchen muss das medizinische Fachpersonal eine Schlüsselrolle beim Schutz gefährdeter Mädchen übernehmen. Wenn eine beschnittene Frau ein Mädchen zur Welt bringt, sollten medizinische Fachpersonen unbedingt das Gespräch mit ihr und ihrem Mann suchen, sie über die gesundheitlichen Folgen unterrichten und darauf hinweisen, dass die Praktik in der Schweiz verboten ist und strafrechtlich verfolgt wird. Dabei ist es empfehlenswert, interkulturelle Vermittlerinnen beizuziehen. Ebenfalls hilfreich kann das Abgeben von Unterlagen - wie beispielsweise der Präventionsbroschüre «Wir schützen unsere Töchter» von TERRE DES FEMMES Schweiz - sein [12].

Medizinisches Fachpersonal, ausgestattet mit transkulturellen Kompetenzen und spezifischem Wissen, kann also einen wichtigen Beitrag zur Überwindung von FGM in der Schweiz leisten. So dass in Zukunft kein Mädchen mehr durch Verstümmelung an seinen Genitalien seiner fundamentalen Rechte beraubt wird. 